\title{
Distinctive Representation of Mispredicted and Unpredicted Prediction Errors in Human Electroencephalography
}

\author{
Yi-Fang Hsu, ${ }^{1,2,3}$ Solene Le Bars, ${ }^{1,2}$ Jarmo A. Hämäläinen, ${ }^{4}$ and Florian Waszak ${ }^{1,2}$ \\ ${ }^{1}$ Université Paris Descartes, Sorbonne Paris Cité, 75006 Paris, France, ${ }^{2}$ Centre National de la Recherche Scientifique, Laboratoire Psychologie de la \\ Perception, Unité Mixte de Recherche 8242, 75006 Paris, France, ${ }^{3}$ Department of Educational Psychology and Counselling, National Taiwan Normal

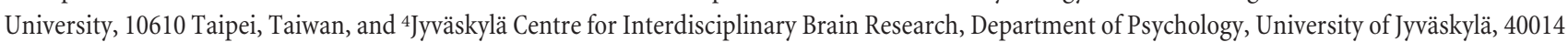 \\ Jyväskylä, Finland
}

The predictive coding model of perception proposes that neuronal responses are modulated by the amount of sensory input that the internal prediction cannot account for (i.e., prediction error). However, there is little consensus on what constitutes nonpredicted stimuli. Conceptually, whereas mispredicted stimuli may induce both prediction error generated by prediction that is not perceived and prediction error generated by sensory input that is not anticipated, unpredicted stimuli involves no top-down, only bottom-up, propagation of information in the system. Here, we examined the possibility that the processing of mispredicted and unpredicted stimuli are dissociable at the neurophysiological level using human electroencephalography. We presented participants with sets of five tones in which the frequency of the fifth tones was predicted, mispredicted, or unpredicted. Participants were required to press a key when they detected a softer fifth tone to maintain their attention. We found that mispredicted and unpredicted stimuli are associated with different amount of cortical activity, probably reflecting differences in prediction error. Moreover, relative to predicted stimuli, the mispredicted prediction error manifested as neuronal enhancement and the unpredicted prediction error manifested as neuronal attenuation on the N1 event-related potential component. These results highlight the importance of differentiating between the two nonpredicted stimuli in theoretical work on predictive coding.

Significance Statement

The current research seeks to dissociate the neurophysiological processing of two types of "nonpredicted" stimuli that have long been considered interchangeable: mispredicted and unpredicted stimuli. We found that mispredicted stimuli, which violate predictions, and unpredicted stimuli, which lack predictions, are represented distinctively in the brain. The results will influence the design of experiments on the predictive coding mechanism, in which the contrast between predicted and "nonpredicted" conditions should be specifically defined to reveal the prediction error proper. This is of general interest because it concerns the logic of research investigating all levels of processing (including perceptual, motor, and cognitive processing) in many neuroscientific domains.

\section{Introduction}

The predictive coding model of perception postulates that perception entails two distinct neurocomputational components, the top-down propagation of prediction and the bottom-up propagation of prediction error (Rao and Ballard, 1999; Friston, $2005,2009)$. Specifically, the flow of information takes place between multiple hierarchical levels harboring both representational units and error units (Egner et al., 2010; Arnal and Giraud, 2012). While the representational units encode prediction about the causal structure of the environment and feed it backward to the next lower level, the error units encode sensory input that prediction fails to explain as prediction error and communicate it forward to the next higher level. Such prediction error is believed 
to modulate a range of neuronal responses (den Ouden et al., 2012).

Within this framework, as recently pointed out by Arnal and Giraud (2012), there is a conceptual difference between mispredicted and unpredicted stimuli (Fig. 1A). In particular, they proposed that mispredicted stimuli are associated with larger prediction error because prediction fails to match with sensory input, inducing both prediction error generated by prediction that is not perceived and prediction error generated by sensory input that is not anticipated. Conversely, unpredicted stimuli are associated with smaller prediction error because there is only the latter type of prediction error: sensory input that is not anticipated. Meanwhile, predicted stimuli are associated with minimum prediction error because prediction explains away sensory input.

Note that this reasoning presupposes that the representation of prediction is discrete-that is, precisely defined. In this case, a correct prediction perfectly matches with the sensory input, canceling out the bottom-up flow of information. However, it is possible that prediction is not represented in an all-or-none manner. Instead of predicting exclusively the most likely upcoming event, the brain is more likely to represent prediction as a probability distribution (Friston, 2009) in one or both of the following ways. First, the representation of prediction might be based on codes that are to a certain degree imprecise in the sense that the activation in each representational unit is distributed over a set of a continuum of predictions. This would be similar to the population coding scheme involving neurons with a Gaussian tuning curve in representing sensory input (Bitterman et al., 2008). Here, predicted stimuli trigger prediction error to the extent that the probability function representing the prediction is wider than the probability function representing the sensory input (Fig. 1B). Second, the representation of prediction might mimic the regularities of the environment, which not only codes the most likely upcoming event, but also other events to a lesser degree. Therefore, when a "predicted" stimulus is presented, there is always some amount of prediction error resulting from the unfulfilled part of the prediction (Fig. 1C).

In both of the aforementioned scenarios, predicted stimuli should trigger medium prediction error because prediction is represented as a probability distribution that inevitably induces some amount of prediction error. Therefore, although both mispredicted and unpredicted stimuli trigger prediction error, their relation to predicted stimuli may be distinct. Compared with predicted stimuli, mispredicted prediction error would manifest as neuronal enhancement, whereas unpredicted prediction error would manifest as neuronal attenuation given the absence of prediction in this case.

Here, we presented participants with sets of five tones in which the frequency of the fifth tones was predicted, mispredicted, or unpredicted and assessed the ensuing prediction error response with electroencephalography (EEG). If the processing of mispredicted and unpredicted stimuli are indeed different, they should be associated with different amount of cortical activ- predicted mispredicted

unpredicted
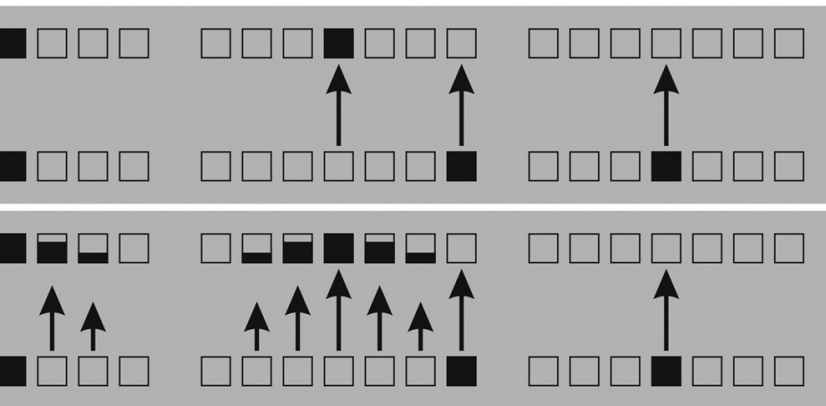

$\square \square \square \square \square$
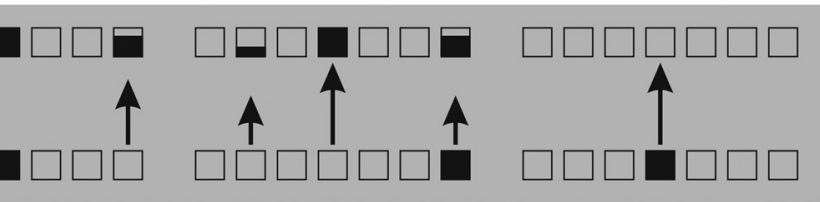

ity, particularly in relation to the $\mathrm{N} 1$ event-related potential (ERP) component, which is typically considered an electrophysiological indicator for automatic predictive processing (for review, see Bendixen et al., 2012). Moreover, if predictions are represented in a probabilistic manner, the amount of cortical activity triggered by predicted stimuli should be in between the cortical activity to mispredicted and unpredicted stimuli.

\section{Materials and Methods}

\section{Participants}

Twenty-one healthy volunteers (average age 24; seven males; 15 righthanded) with no history of neurological, psychiatric, or visual/hearing impairments as indicated by self-report participated in the experiment. Participants gave written informed consent and were paid for participation. Ethical approval was granted by the Comité de Protection des Personnes Ile de France II. One participant was excluded from data analysis for having $<50 \%$ artifact-free trials in any of the conditions of interest, leaving 20 participants in the final sample (average age 25; seven males; 14 right-handed).

\section{Stimuli}

Seven sinusoidal tones with a loudness of 80 phons (i.e., $80 \mathrm{~dB}$ for tones of $1000 \mathrm{~Hz}$ ) were generated using MATLAB. The duration of each tone was $50 \mathrm{~ms}$ (including $5 \mathrm{~ms}$ rise/fall times). The frequency of each tone was within the range of $261.626-493.883 \mathrm{~Hz}$, matching the absolute frequency of a series of seven natural keys on a modern piano (i.e., C4 D4 E4 F4 G4 A4 B4).

From the pool of seven tones, a total of 800 sets of five tones were created for the main experiment. Figure 2 shows a schematic illustration of the design. In the predicted condition, which comprised 600 sets of tones, the frequency of the first four tones was ascending in steps of one natural key and the frequency of the fifth tone was one natural key higher than the fourth tone in the series (e.g., D4-E4-F4-G4-A4). That is, the fifth tone followed the ascending pattern. In the mispredicted condition, which comprised 100 sets of tones, the frequency of the first four tones was ascending in steps of one natural key and the frequency of the fifth tone was one natural key lower than the first tone in the series (e.g., D4-E4-F4-G4-C4); that is, the fifth tone violated the ascending pattern. In the unpredicted condition, which comprised 100 sets of tones, stimuli 


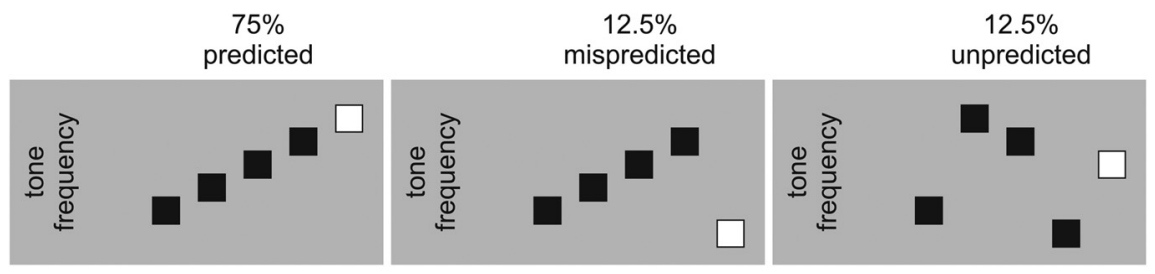

Figure 2. Schematicillustration of the design. In the experiment, $75 \%$ of the trials have a predicted fifth tone, $12.5 \%$ of the trials have a mispredicted fifth tone, and the last $12.5 \%$ of the trials have an unpredicted fifth tone.

were created by randomly scrambling the order of the five tones in the predicted and mispredicted conditions. Here, the fifth tone cannot be predicted because the five tones in each sequence were selected from a population of seven tones.

To exclude the possibility that the findings in the main experiment simply reflect how much the tones of interest (i.e., the fifth tones) differ from their preceding tones (i.e., the fourth tones) in terms of frequency, a total of 160 sets of two tones were created for a control experiment. Half of the two-tone sets resemble the fourth and the fifth tones in the predicted condition in the main experiment; that is, the second tones were always one natural key higher than the first tones (e.g., G4-A4). The other half of the two-tone sets resemble the fourth and the fifth tones in the mispredicted condition in the main experiment; that is, the second tones were always four natural key lower than the first tones (e.g., G4C4). In other words, the frequency difference between the two tones in the control experiment was identical to the frequency difference between the fourth and the fifth tones in the main experiment. However, in the control experiment, the second tones being the one-natural-key-higher tones and the four-natural-key-lower tones are of equal probability and thus equal predictability.

E-prime version 2.0 (Psychology Software Tools) was used for stimulus presentation. The stimulation was randomized individually for each participant and delivered binaurally via headphones (Sennheiser PX200).

\section{Procedures}

In the main experiment, a total of 10 blocks of 80 trials were presented. A trial started with the presentation of a fixation cross, which remained on the screen for the duration of the trial. After $500 \mathrm{~ms}$, a set of five tones were presented and each tone was followed by a $500 \mathrm{~ms}$ stimulus onset asynchrony (SOA). Ten percent of the fifth tones were of attenuated loudness by $20 \mathrm{~dB}$ in each condition. To maintain their attention, participants were required to press a key when they detected a softer fifth tone within a $700 \mathrm{~ms}$ long time window after the offset of the fifth tone. Each trial was followed by a jittered intertrial interval (ITI) of $700-800 \mathrm{~ms}$.

In the control experiment, a total of two blocks of 80 trials were presented. A trial started with the presentation of a fixation cross, which remained on the screen for the duration of the trial. After $500 \mathrm{~ms}$, a set of two tones were presented in which each tone was followed by a $500 \mathrm{~ms}$ SOA. Ten percent of the second tones were of attenuated loudness by 20 $\mathrm{dB}$ in each condition. To maintain their attention, participants were required to press a key when they detected a softer second tone within a $700 \mathrm{~ms}$ long time window after the offset of the second tone. Each trial was followed by a jittered ITI of 700-800 ms.

To prevent the main experiment from influencing the control experiment, participants were presented with the control experiment first. The whole study took $\sim 60 \mathrm{~min}$ (i.e., 160 trials $\times 2500 \mathrm{~ms}+800$ trials $\times 4000 \mathrm{~ms}$ ).

\section{Data recording and analysis}

EEG recording and preprocessing. EEG was recorded with 64 active electrodes (actiCAP; Brain Products) conforming to the international 10-10 system. The sampling rate was $500 \mathrm{~Hz}$. No online/offline filter was used. The $\mathrm{Cz}$ served as the reference electrode online. The data were recomputed to average reference offline. Target stimuli of attenuated loudness were removed. Epochs extended from -100 to 500 ms relative to the onset of the last stimulus in each trial (i.e., the fifth stimulus in the main experiment and the second stimulus in the control experiment) using a $100 \mathrm{~ms}$ prestimulus baseline. Ocular artifact correction was conducted with independent component analysis in EEGlab (Delorme and Makeig, 2004). Epochs containing voltage deviations exceeding $\pm 100 \mu \mathrm{V}$ relative to baseline at any of the electrodes were rejected. The trial numbers after artifact rejection in each condition are listed in Table 1.

ERP analysis. ERP analysis was based on a temporal principal component analysis (PCA) in SPSS 20. Since it was first introduced (Ruchkin et al., 1964; Donchin, 1966), PCA has been considered an effective linear reduction method for multivariate ERP data (Möcks, 1988a, 1988b; Duffy et al., 1992; Chapman and McCrary, 1995; Dien, 1998; Picton et al., 2000; Dien and Frishkoff, 2005; for review, see Kayser and Tenke, 2003, Dien, 2012). PCA statistically decomposes the ERP waveforms into constituent building blocks, which affords objective, data-driven ERP component measures compared with other conventional methods (Kayser et al., 1998; Beauducel et al., 2000; Kayser and Tenke, 2006). Moreover, it is not as susceptible to the influences of high-frequency noises and low-frequency drifts in the data as other conventional methods (Luck, 2005). Covariance matrix and Promax rotation were used here. All components accounting for a total of $99 \%$ of the variance (maximum iterations for convergence $=500)$ were included in the rotation $($ Promax kappa $=4)$. The decomposition provided a set of time-variant component loadings reflecting the contribution of each temporal component to the voltage at each time point and a set of time-invariant component scores (calculated using Bartlett method) representing the contribution of each temporal component to the ERP waveforms which can be subject to inferential statistics (Van Boxtel, 1998). The components corresponding to the N1 (i.e., principal component 2 accounting for $15.59 \%$ of the variance), the N1/P2 transition in which a mismatch negativity (MMN) was seen in mispredicted condition but not in unpredicted condition (i.e., principal component 3 accounting for $6.01 \%$ of the variance), and the P2 (i.e., principal component 4 accounting for $2.87 \%$ of the variance) were identified on the basis of the component loading latencies and the component score topographies.

The component scores were averaged across three electrodes showing the largest responses across all conditions independent of experimental manipulation (i.e., N1: F1, F4, FC3; N1/P2 transition: F3, F5, F4; P2: Pz, $\mathrm{P} 2, \mathrm{CPz})$. Therefore, the averages of these electrodes can be considered as objective representatives of the components to serve as inputs for further statistical analyses. The advantage of averaging three maximum electrodes was twofold. First, it increased the signal-to-noise ratio of the components. Second, it avoided the problems inherited in the analysis of predefined areas that took an average of multiple electrodes over predefined regions, which might not correspond to the true topography in the experiment. Moreover, these electrodes representing the locations of maximum signals for each component corresponded to those reported in the literature (Näätänen and Picton, 1987).

To test for the prediction effects in the main experiment, a one-way repeated-measures ANOVA comparing the ERPs among the predicted, mispredicted, and unpredicted conditions was performed. To test whether the prediction effects in the main experiment simply reflect how much the tones of interest (i.e., the fifth tones) differ from their preceding tones (i.e., the fourth tones) in terms of frequency, a 2 (frequency distance: one-natural-key-higher/four-natural-key-lower conditions) $\times$ 2 (predictive setting: main/control experiments) repeated-measures ANOVA was performed.

Descriptive source analyses of the N1 were performed in BESA Research 6.0 using equivalent current dipoles (ECDs) in a 4-shell ellipsoidal head model (Scherg and Von Cramon, 1986). For each condition, a time window at $92-112 \mathrm{~ms}$ (i.e., $\pm 10 \mathrm{~ms}$ around the peak of the N1) that showed stable topography was chosen for analysis. Two ECDs were fitted without constraints separately for the grand average ERPs of each condition, resulting in a good fit (residual variance for predicted: $1.67 \%$, mispredicted: $2.31 \%$, and unpredicted: $3.52 \%$ conditions). 
Table 1. Mean and range of trial numbers after artefact rejection in each condition

\begin{tabular}{|c|c|c|c|c|c|}
\hline & Main experiment & & & Control experiment & \\
\hline & Predicted & Mispredicted & Unpredicted & One-natural-key-higher & Four-natural-key-lower \\
\hline Mean (range) & $519.60(485-537)$ & $86.95(81-90)$ & $86.20(80-90)$ & $68.70(54-72)$ & $68.40(54-72)$ \\
\hline
\end{tabular}

A

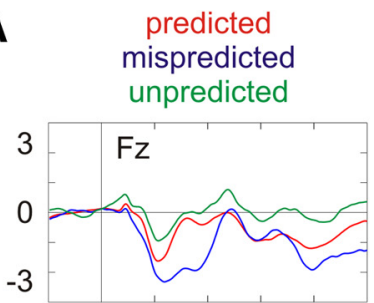

predicted
mispredicted unpredicted
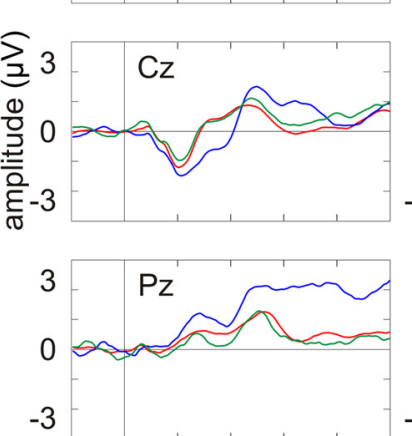

$-1000$ one-natural-key-higher four-natural-key-lower
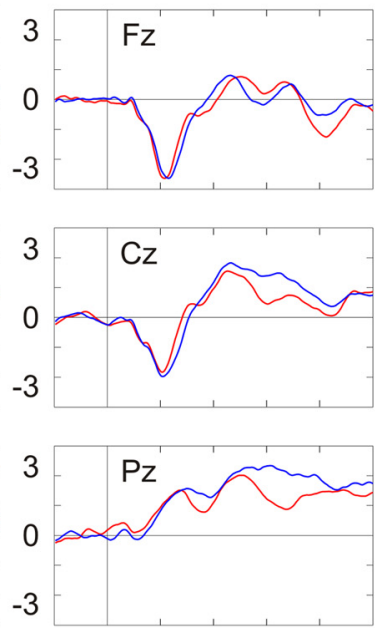

$500-100 \quad 0$

time (ms)

\section{B}

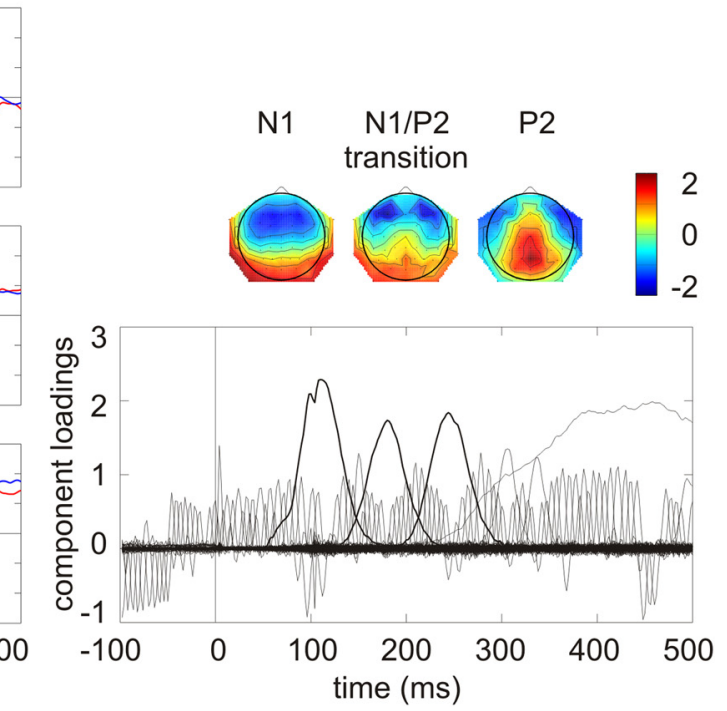

Figure 3. A, Grand average ERPs on three midline electrodes (i.e., $\mathrm{Fz}, \mathrm{Cz}, \mathrm{Pz}$ ). The waveforms were low-pass filtered at $30 \mathrm{~Hz}$ for visual presentation purposes. $\boldsymbol{B}$, Component loadings of 122 components in the temporal PCA for the ERP data across all conditions. The component corresponding to the N1, the N1/P2 transition, and the P2 in the grand average ERPs (i.e., principal component 2,3, and 4, respectively) are marked with thick lines with the component score topographies plotted on the top.

\section{Results}

Behavioral results

Participants' target detection performance was close to ceiling in the main experiment [hit rate: mean $=0.97, \mathrm{SD}=0.04$; false alarm rate: mean $<0.001, \mathrm{SD} \leq 0.001$; reaction time $(\mathrm{RT})$ : mean $=419.29, \mathrm{SD}=55.19]$ and in the control experiment (hit rate: mean $=0.94, \mathrm{SD}=0.12$; false alarm rate: mean $=0.05$, $\mathrm{SD}=0.07 ; \mathrm{RT}:$ mean $=404.49, \mathrm{SD}=71.70)$, indicating that they concentrated on the stimuli as instructed.

\section{ERP results}

Figure $3 A$ shows the grand average ERPs on three midline electrodes (i.e., $\mathrm{Fz}, \mathrm{Cz}, \mathrm{Pz}$ ). The waveforms were low-pass filtered at $30 \mathrm{~Hz}$ for visual presentation purposes. There are evident N1 at $\sim 100 \mathrm{~ms}$ with a frontocentral distribution and P2 at $\sim 250$ ms with a centroparietal distribution. Figure $3 B$ shows the component loadings of 122 components in the temporal PCA. The components corresponding to the N1, the $\mathrm{N} 1 / \mathrm{P} 2$ transition, and the $\mathrm{P} 2$ in the grand average ERPs were identified on the basis of the component loading latencies and the component score topographies.

\section{Comparisons among the predicted, mispredicted, and} unpredicted conditions

On the N1, there was a significant difference among the predicted, mispredicted, and unpredicted conditions $\left(F_{(2,38)}=\right.$ $36.77, p<0.001$, partial $\left.\eta^{2}=0.66\right)$. Post hoc pairwise comparisons with Sidak adjustment showed that the N1 was significantly larger in the mispredicted than in the predicted condition (mean difference $=-0.42$; SE $=0.08 ; p<0.001 ; 95 \% \mathrm{CI}=-0.62-$ -0.22 ) and significantly smaller in the unpredicted than in the predicted condition (mean difference $=0.20 ; \mathrm{SE}=0.07 ; p<$ 0.025; 95\% CI = 0.02-0.37) (Fig. 4A, left column).

Descriptive source analysis using ECDs showed that the largest contribution to the $\mathrm{N} 1$ response in each condition originated near the left and right auditory cortices (Fig. 5). No additional cortical sources were identified with stable solutions.

On the N1/P2 transition, there was a significant difference among the predicted, mispredicted, and unpredicted conditions $\left(F_{(2,38)}=15.37, p<0.001\right.$, partial $\left.\eta^{2}=0.45\right)$. Post hoc pairwise comparisons with Sidak adjustment showed that the N1/P2 transition was significantly larger in the mispredicted than in the predicted condition (mean difference $=-0.54 ; \mathrm{SE}=0.14 ; p<$ $0.01 ; 95 \% \mathrm{CI}=-0.92--0.17$ ), but did not differ between the unpredicted and the predicted conditions (mean difference $=$ $0.15 ; \mathrm{SE}=0.11 ; p=0.46 ; 95 \% \mathrm{CI}=-0.14-0.43$; Fig. $4 \mathrm{~A}$, middle column).

On the $\mathrm{P} 2$, there was a significant difference among the predicted, mispredicted, and unpredicted conditions $\left(F_{(2,38)}=4.51\right.$, $p<0.05$, partial $\left.\eta^{2}=0.19\right)$. Post hoc pairwise comparisons with Sidak adjustment showed that the P2 was marginally larger in the mispredicted than in the predicted condition (mean difference $=$ 0.36 ; $\mathrm{SE}=0.12 ; p=0.026 ; 95 \% \mathrm{CI}=0.04-0.67)$, but did not differ between the unpredicted and predicted conditions (mean difference $=0.05 ; \mathrm{SE}=0.12 ; p=0.96 ; 95 \% \mathrm{CI}=-0.25-0.36$; Fig. $4 A$, right column).

Interactions between frequency distance and predictive setting To test whether the prediction effects in the main experiment simply reflect how much the tones of interest (i.e., the fifth tones) differ from their preceding tones (i.e., the fourth tones) in terms 

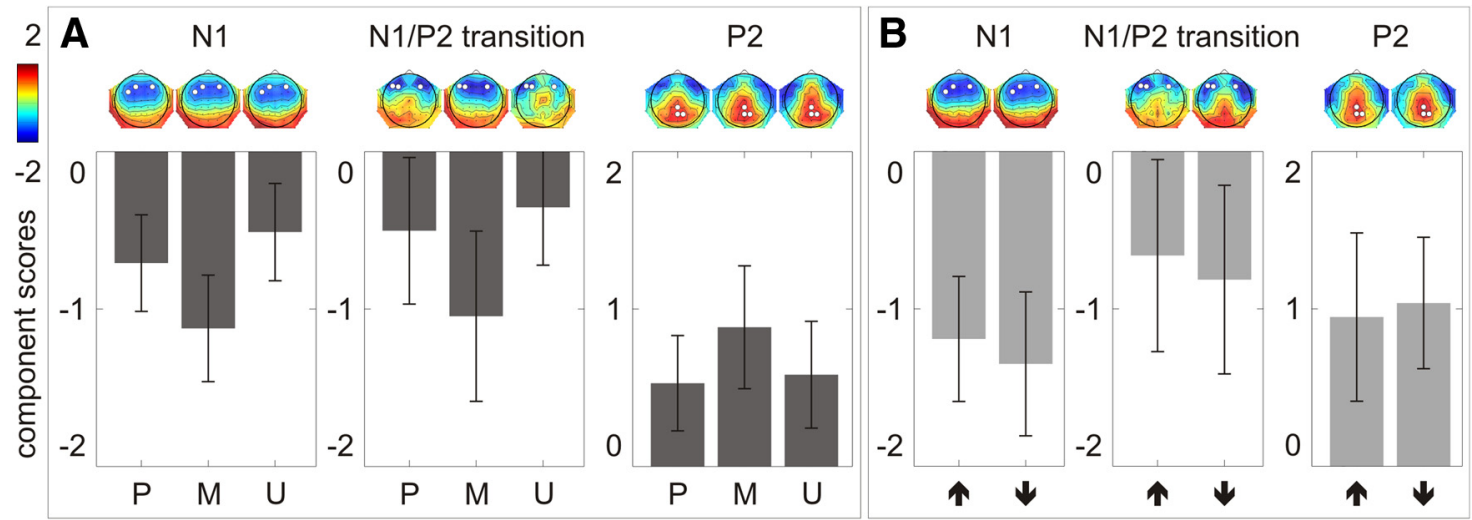

Figure 4. The N1,N1/P2 transition, and P2 component scores averaged across three electrodes showing the largest response (marked as white dots on the component score topographies) in each condition. $\boldsymbol{A}$, In the main experiment, comparisons between predicted $(\mathrm{P})$, mispredicted $(\mathrm{M})$, and unpredicted $(\mathrm{U})$ conditions are illustrated. $\boldsymbol{B}$, In the control experiment, comparisons between one-natural-key-higher (upward arrow) and four-natural-key-lower (downward arrow) conditions are illustrated. Error bars depict one SD of the mean.

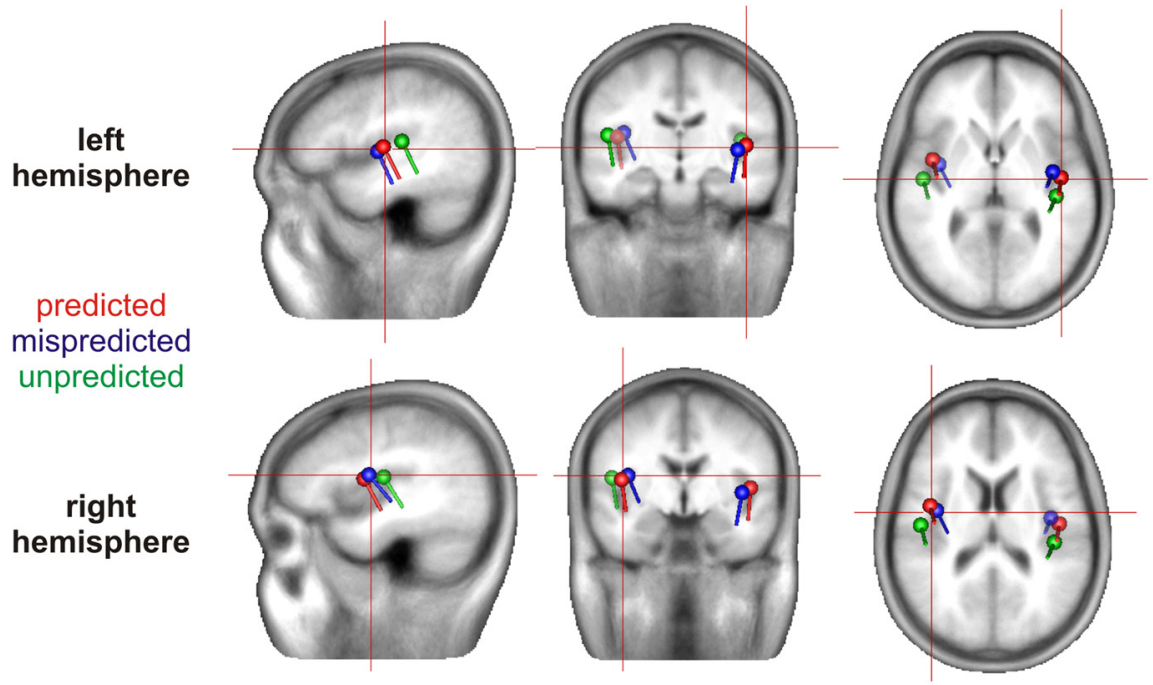

Figure 5. ECDs localization of the N1 cortical activity based on the grand average ERPs in the predicted, mispredicted, and unpredicted conditions.

of frequency, data from the main experiment (Fig. 4A) and those from the control experiment (Fig. $4 B$ ) were conjointly submitted to a 2 (frequency distance: one-natural-key-higher/four-naturalkey-lower conditions) $\times 2$ (predictive setting: main/control experiments) repeated-measures ANOVA.

On the N1, there was a significant interaction between frequency distance and predictive setting $\left(F_{(1,19)}=6.26, p<0.05\right.$, partial $\left.\eta^{2}=0.25\right)$. Post hoc $t$ tests showed that there was a significant difference between frequency distance in the main experiment $\left(t_{(19)}=5.42, p<0.001\right)$, but not in the control experiment $\left(t_{(19)}=1.74, p=0.10\right)$.

On the N1/P2 transition, there was a significant interaction between frequency distance and predictive setting $\left(F_{(1,19)}=6.35\right.$, $p<0.05$, partial $\left.\eta^{2}=0.25\right)$. Post hoc $t$ tests showed that there was a significant difference between frequency distance in the main experiment $\left(t_{(19)}=3.82, p \leq 0.001\right)$, but not in the control experiment $\left(t_{(19)}=0.85, p=0.41\right)$.

On the P2, there was no significant interaction between frequency distance and predictive setting $\left(F_{(1,19)}=1.64, p=0.22\right.$, partial $\left.\eta^{2}=0.08\right)$. Conversely, there was a significant main effect of frequency distance in which the P2 was smaller in the smallfrequency-distance condition than in the large-frequency- distance condition $\left(F_{(1,19)}=4.95, p<\right.$ 0.05 , partial $\eta^{2}=0.21$ ) and a significant main effect of predictive setting in which the $\mathrm{P} 2$ was smaller in the main experiment than in the control experiment $\left(F_{(1,19)}=\right.$ 6.90, $p<0.05$, partial $\left.\eta^{2}=0.27\right)$.

Overall, the significant interactions between frequency distance and predictive setting on the N1 (and even the N1/P2 transition but not the P2) excludes the possibility that the prediction effects in the main experiment simply reflect how much the tones of interest (i.e., the fifth tones) differ from their preceding tones (i.e., the fourth tones) in terms of frequency.

\section{Discussion}

We presented participants with sets of five tones, the last of which was predicted, mispredicted, or unpredicted in terms of frequency. We found that mispredicted and unpredicted stimuli are associated with different amounts of cortical activity, probably reflecting differences in prediction error (Friston, 2005; den Ouden et al., 2012). In particular, predicted stimuli did not trigger minimum, but rather medium cortical activity. Relative to predicted stimuli, mispredicted stimuli are associated with neuronal enhancement and unpredicted stimuli are associated with neuronal attenuation on the N1 ERP component. Source localization suggested that the predictive model is generated near the auditory cortices.

First of all, our results demonstrate that the processing of mispredicted and unpredicted stimuli are distinctive. The dissociation conforms to previous research on the MMN reporting a significant "surprise response" by contrasting between a deviant embedded in a standard sequence (i.e., a mispredicted tone) and a deviant embedded in an equiprobable sequence (i.e., an unpredicted tone) (for review, see Jacobsen and Schröger, 2001; Näätänen et al., 2005; but see Ahmed et al., 2011; Astikainen et al., 2011; Nakamura et al., 2011 vs Farley et al., 2010; Fishman and Steinschneider, 2012; Kaliukhovich and Vogels, 2014 for an ongoing debate in animal research). Importantly, in addition, our results demonstrate how mispredicted and unpredicted responses are related to predicted response, which can help to 
explain the seemingly contradictory observations of predictionrelated effects in the literature. For example, although stimulus repetition is believed to induce prediction (Summerfield et al., 2008), the nonpredicted alternation of the familiar stimuli was associated with neuronal enhancement, whereas the nonpredicted alternation of the unfamiliar stimuli was associated with neuronal attenuation (Henson et al., 2000; Fiebach et al., 2005; Gagnepain et al., 2008; Soldan et al., 2008; Müller et al., 2013; Subramaniam et al., 2012). Notably, familiar and unfamiliar stimuli differ in whether there is a preexisting representation (Turk-Browne et al., 2008), which might determine whether a prediction can be encoded in the representational units (Grotheer and Kovács, 2014). It might be that the nonpredicted alternation of the familiar and unfamiliar stimuli were like the mispredicted and unpredicted case, respectively. More closely related to the current research, Hsu et al. (2014a, 2014b) also found seemingly contradictory patterns of activation in two auditory studies using very similar designs. When pairs of two tones were contrasted with each other, more enhanced N1 responses were found for the alternated tones (i.e., the nonpredicted tones) than the repeated tones (i.e., the predicted tones) (Hsu et al., 2014a). Conversely, when pairs of random tones and pairs of rising tones were contrasted, more attenuated N1 responses were found for the random pairs (i.e., the nonpredicted tones) than the rising pairs (i.e., the predicted tones) (Hsu et al., 2014b). The two results seem incompatible when both alternated and random tones are simply considered as "nonpredicted" stimuli. However, the pattern can be explained if alternated and random tones are, respectively, considered as mispredicted and unpredicted stimuli. Specifically, in the former study, alternated tones were embedded in pairs of two identical tones in which there was perceptual regularity. This might facilitate the brain's attempt to form prediction. In this case, alternated tones would be more like mispredicted stimuli, which trigger larger cortical activity relative to predicted stimuli. Conversely, in the latter study, random tones were embedded in a stimulus stream without perceptual regularity and there was little if any prediction involved. In this case, random tones would be more like unpredicted stimuli, which trigger smaller cortical activity relative to predicted stimuli.

The pattern of results is straightforward as concerns the mispredicted stimuli. Relative to predicted stimuli, mispredicted stimuli are associated with enhanced prediction error. This is consistent with a range of findings showing that violation of prediction triggers stronger cortical activity (for review, see Bendixen et al., 2012). The common explanation for this is that, for mispredicted stimuli, prediction fails to match with sensory input. This mismatch therefore induces both prediction error generated by prediction that is not perceived and prediction error generated by sensory input that is not anticipated (Arnal and Giraud, 2012). This idea was also used to explain why a decrease of stimulus repetition probability reduces the size of adaptation effect on hemodynamic responses (visual: Summerfield et al., 2008; Kovács et al., 2012; Grotheer and Kovács, 2014; Mayrhauser et al., 2014; auditory: Andics et al., 2013) and electrophysiological responses (visual: Summerfield et al., 2011; auditory: Todorovic et al., 2011). Specifically, it was proposed that our perceptual apparatus generally predicts stimulation to be consistent from moment to moment because our perceptual context tends to remain stable across time scales (Summerfield et al., 2008). In this manner, a decrease of stimulus repetition probability is considered a violation of prediction, which in turn elicits stronger cortical activity.
A different pattern of results was observed for the unpredicted stimuli. Relative to predicted stimuli, unpredicted stimuli are associated with attenuated prediction error. This is incompatible with the idea that predicted stimuli are associated with minimum prediction error because a fulfilled prediction can explain away all the prediction error (Arnal and Giraud, 2012). Rather, predicted stimuli are associated with some amount of prediction error. This finding provides empirical support for the neurocomputational theory that, instead of encoding prediction in an allor-none manner, the brain seems to represent prediction as a probability distribution (Friston, 2009) in one or both of the following ways.

First, the representation of prediction might be based on codes that are to a certain degree imprecise in the sense that the activation in each representational unit is distributed over a set of a continuum of predictions. With regard to continuous variables such as frequency, the prediction of the "preferred" frequency would be coded most strongly. This is done similarly to the population coding scheme involving neurons with a Gaussian tuning curve in representing sensory input (Bitterman et al., 2008). Here, predicted stimuli trigger prediction error to the extent that the probability function representing the prediction is wider than the probability function representing the sensory input. Second, the representation of prediction might mimic the regularities of the environment. In the current research, participants might learn that, after four ascending tones, the chance that the step inertia will continue is $86 \%$ and the chance that the step inertia will change is $14 \%$. Although the former case is considered the most likely upcoming event, the latter case is also coded in the representational units, only to a lesser degree. Therefore, when a "predicted" stimulus is presented, there is always some amount of prediction error resulting from the unfulfilled part of the prediction. In both of the aforementioned scenarios, which are not mutually exclusive, predicted stimuli trigger some amount of prediction error that is larger or at least equivalent to the unpredicted prediction error.

Note that, in the current research, all stimuli were attended. Can our result pattern be attributed to attention effect? It was suggested the prediction mechanism dynamically interacts with other cognitive operations, particularly attention (Summerfield and Egner, 2009; Segaert et al., 2013). Specifically, the engagement of attention may increase the weighting of sensory information according to the precision of prediction, resulting in heightened cortical activity to predicted relative to nonpredicted stimuli (Rao, 2005; Feldman and Friston, 2010; Kok et al., 2012). However, this idea cannot explain the distinct pattern of cortical activity to mispredicted and unpredicted stimuli reported here, both of which are supposed to be nonpredicted stimuli of low precision of prediction. Conversely, the result pattern might be interpreted in terms of how prediction error can be adjusted depending on the predictability of the input (Friston, 2009). Specifically, prediction error evoked by stimuli occurring in more predictable contexts can be weighted more strongly (Schröger et al., 2015). Here, because the preceding contextual stimuli were more predictable for mispredicted stimuli than unpredicted stimuli, neuronal responses were stronger in the former than the latter. Altogether, the current research suggests that it is necessary to differentiate between the two nonpredicted stimuli in theoretical work on predictive coding.

To summarize, the predictive coding model of perception proposes that neuronal responses reflect the sensory input that the prediction cannot account for (Rao and Ballard, 1999; Friston, 2005, 2009). However, it remains unclear what constitutes 
the nonpredicted stimuli. Although a conceptual difference between mispredicted and unpredicted stimuli was proposed (Arnal and Giraud, 2012), there is a lack of empirical investigation on this issue. Here, we examined this possibility that the processing of mispredicted and unpredicted stimuli are dissociable at the neurophysiological level. We found that, compared with predicted stimuli, mispredicted stimuli elicited neuronal enhancement, whereas unpredicted stimuli elicited neuronal attenuation. This pattern of results demonstrates the essentialness to differentiate between mispredicted and unpredicted stimuli. Moreover, it shows that predicted stimuli are not necessarily associated with minimum cortical activity. We suggest that prediction is rather represented as a probability distribution, resulting in partially explained and unexplained sensory input and thus medium prediction error.

\section{References}

Ahmed M, Mällo T, Leppänen PH, Hämäläinen J, Ayräväinen L, Ruusuvirta T, Astikainen P (2011) Mismatch brain response to speech sound changes in rats. Front Psychol 2:283. Medline

Andics A, Gál V, Vicsi K, Rudas G, Vidnyánszky Z (2013) fMRI repetition suppression for voices is modulated by stimulus expectations. Neuroimage 69:277-283. CrossRef Medline

Arnal LH, Giraud AL (2012) Cortical oscillations and sensory predictions. Trends Cogn Sci 16:390-398. CrossRef Medline

Astikainen P, Stefanics G, Nokia M, Lipponen A, Cong F, Penttonen M, Ruusuvirta T (2011) Memory-based mismatch response to frequency changes in rats. PLoS One 6(9).

Beauducel A, Debener S, Brocke B, Kayser J (2000) On the reliability of augmenting/reducing: peak amplitudes and principal components analysis of auditory evoked potentials. Journal of Psychophysiology 14:226240. CrossRef

Bendixen A, SanMiguel I, Schröger E (2012) Early electrophysiological indicators for predictive processing in audition: a review. Int J Psychophysiol 83:120-131. CrossRef Medline

Bitterman Y, Mukamel R, Malach R, Fried I, Nelken I (2008) Ultra-fine frequency tuning revealed in single neurons of human auditory cortex. Nature 451:197-201. CrossRef Medline

Chapman RM, McCrary JW (1995) EP component identification and measurement by principal components analysis. Brain Cogn 27:288-310. CrossRef Medline

Delorme A, Makeig S (2004) EEGLAB: an open source toolbox for analysis of single-trial EEG dynamics including independent component analysis. J Neurosci Methods 134:9-21. CrossRef Medline

den Ouden HE, Kok P, de Lange FP (2012) How prediction errors shape perception, attention, and motivation. Front Psychol 3:548. Medline

Dien J (1998) Addressing misallocation of variance in principal components analysis of event-related potentials. Brain Topography 11:43-55. CrossRef Medline

Dien J (2012) Applying principal components analysis to event-related potentials: a tutorial. Dev Neuropsychol 37:497-517. CrossRef Medline

Dien J, Frishkoff GA (2005) Principal components analysis of event-related potential datasets. In: Event-related potentials: a methods handbook (Handy T, ed), pp 189-208. Cambridge, MA: MIT.

Donchin E (1966) A multivariate approach to the analysis of average evoked potentials. IEEE Trans Biomed Eng 13:131-139. Medline

Duffy FH, Jones K, Bartels P, McAnulty G, Albert M (1992) Unrestricted principal components analysis of brain electrical activity: issues of data dimensionality, artifact, and utility. Brain Topography 4:291-307. CrossRef Medline

Egner T, Monti JM, Summerfield C (2010) Expectation and surprise determine neural population responses in the ventral visual stream. J Neurosci 30:16601-16608. CrossRef Medline

Farley BJ, Quirk MC, Doherty JJ, Christian EP (2010) Stimulus-specific adaptation in auditory cortex is an NMDA-independent process distinct from the sensory novelty encoded by the mismatch negativity. J Neurosci 30:16475-16484. CrossRef Medline

Feldman H, Friston KJ (2010) Attention, uncertainty, and free-energy. Front Hum Neurosci 4:215. Medline

Fiebach CJ, Gruber T, Supp GG (2005) Neuronal mechanisms of repetition priming in occipitotemporal cortex: spatiotemporal evidence from functional magnetic resonance imaging and electroencephalography. J Neurosci 25:3414-3422. CrossRef Medline

Fishman YI, Steinschneider M (2012) Searching for the mismatch negativity in primary auditory cortex of the awake monkey: deviance detection or stimulus specific adaptation? J Neurosci 32:15747-15758. CrossRef Medline

Friston K (2005) A theory of cortical responses. Philos Trans R Soc Lond B Biol Sci 360:815-836. CrossRef Medline

Friston K (2009) The free-energy principle: a rough guide to the brain? Trends Cogn Sci 13:293-301. CrossRef Medline

Gagnepain P, Chételat G, Landeau B, Dayan J, Eustache F, Lebreton K (2008) Spoken word memory traces within the human auditory cortex revealed by repetition priming and functional magnetic resonance imaging. J Neurosci 28:5281-5289. CrossRef Medline

Grotheer M, Kovács G (2014) Repetition probability effects depend on prior experiences. J Neurosci 34:6640-6646. CrossRef Medline

Henson R, Shallice T, Dolan R (2000) Neuroimaging evidence for dissociable forms of repetition priming. Science 287:1269-1272. CrossRef Medline

Hsu YF, Hämäläinen JA, Waszak F (2014a) Repetition suppression comprises both attention-independent and attention-dependent processes. Neuroimage 98:168-175. CrossRef Medline

Hsu YF, Hämäläinen JA, Waszak F (2014b) Both attention and prediction are necessary for adaptive neuronal tuning in sensory processing. Front Hum Neurosci 8:152. Medline

Jacobsen T, Schröger E (2001) Is there pre-attentive memory-based comparison of pitch? Psychophysiology 38:723-727. CrossRef Medline

Kaliukhovich DA, Vogels R (2014) Neurons in macaque inferior temporal cortex show no surprise response to deviants in visual oddball sequences. J Neurosci 34:12801-12815. CrossRef Medline

Kayser J, Tenke CE (2003) Optimizing PCA methodology for ERP component identification and measurement: theoretical rationale and empirical evaluation. Clin Neurophysiol 114:2307-2325. CrossRef Medline

Kayser J, Tenke CE (2006) Consensus on PCA for ERP data, and sensibility of unrestricted solutions. Clin Neurophysiol 117:695-707.

Kayser J, Tenke CE, Bruder GE (1998) Dissociation of brain ERP topographies for tonal and phonetic oddball tasks. Psychophysiology 35:576590. CrossRef Medline

Kok P, Rahnev D, Jehee JF, Lau HC, de Lange FP (2012) Attention reverses the effect of prediction in silencing sensory signals. Cereb Cortex 22: 2197-2206. CrossRef Medline

Kovács G, Iffland L, Vidnyánszky Z, Greenlee MW (2012) Stimulus repetition probability effects on repetition suppression are position invariant for faces. Neuroimage 60:2128-2135. CrossRef Medline

Luck SJ (2005) An introduction to the event-related potential technique. Cambridge, MA: MIT.

Mayrhauser L, Bergmann J, Crone J, Kronbichler M (2014) Neural repetition suppression: evidence for perceptual expectation in object-selective regions. Front Hum Neurosci 8(225).

Möcks J (1988a) Decomposing event-related potentials: a new topographic components model. Biol Psychol 26:199-215. CrossRef Medline

Möcks J (1988b) Topographic components model for event-related potentials and some biophysical considerations. IEEE Trans Biomed Eng 35: 482-484. CrossRef Medline

Müller NG, Strumpf H, Scholz M, Baier B, Melloni L (2013) Repetition suppression versus enhancement: it's quantity that matters. Cereb Cortex 23:315-322. Medline

Näätänen R, Picton T (1987) The N1 wave of the human electric and magnetic response to sound: A review and an analysis of the component structure. Psychophysiology 24:375-425. CrossRef Medline

Näätänen R, Jacobsen T, Winkler I (2005) Memory-based or afferent processes in mismatch negativity (MMN): a review of the evidence. Psychophysiology 42:25-32. CrossRef Medline

Nakamura T, Michie PT, Fulham WR, Todd J, Budd TW, Schall U, Hunter M, Hodgson DM (2011) Front Psychol 2:367.

Picton TW, Bentin S, Berg P, Donchin E, Hillyard SA, Johnson R Jr, Miller GA, Ritter W, Ruchkin DS, Rugg MD, Taylor MJ (2000) Guidelines for using human event-related potentials to study cognition: recording standards and publication criteria. Psychophysiology 37:127-152. CrossRef Medline 
Rao RP (2005) Bayesian inference and attentional modulation in the visual cortex. Neuroreport 16:1843-1848. CrossRef Medline

Rao RP, Ballard DH (1999) Predictive coding in the visual cortex: a functional interpretation of some extra-classical receptive-field effects. Nat Neurosci 2:79-87. CrossRef Medline

Ruchkin DS, Villegas J, John ER (1964) An analysis of average evoked potentials making use of least mean square techniques. Ann N Y Acad Sci 115:799-826. CrossRef Medline

Scherg M, Von Cramon D (1986) Evoked dipole source potentials of the human auditory cortex. Electroencephalogr Clin Neurophysiol 65:344360. CrossRef Medline

Schröger E, Marzecová A, SanMiguel I (2015) Attention and prediction in human audition: a lesson from cognitive psychophysiology. Eur J Neurosci 41:641-664. CrossRef Medline

Segaert K, Weber K, de Lange FP, Petersson KM, Hagoort P (2013) The suppression of repetition enhancement: a review of fMRI studies. Neuropsychologia 51:59-66. CrossRef Medline

Soldan A, Zarahn E, Hilton HJ, Stern Y (2008) Global familiarity of visual stimuli affects repetition-related neural plasticity but not repetition priming. Neuroimage 39:515-526. CrossRef Medline

Subramaniam K, Faust M, Beeman M, Mashal N (2012) The repetition par- adigm: enhancement of novel metaphors and suppression of conventional metaphors in the left inferior parietal lobe. Neuropsychologia 50 : 2705-2719. CrossRef Medline

Summerfield C, Egner T (2009) Expectation (and attention) in visual cognition. Trends Cogn Sci 13:403-409. CrossRef Medline

Summerfield C, Trittschuh EH, Monti JM, Mesulam MM, Egner T (2008) Neural repetition suppression reflects fulfilled perceptual expectations. Nat Neurosci 11:1004-1006. CrossRef Medline

Summerfield C, Wyart V, Johnen VM, de Gardelle V (2011) Human scalp electroencephalography reveals that repetition suppression varies with expectation. Front Hum Neurosci 5(67).

Todorovic A, van Ede F, Maris E, de Lange FP (2011) Prior expectation mediates neural adaptation to repeated sounds in the auditory cortex: an MEG study. J Neurosci 31:9118-9123. CrossRef Medline

Turk-Browne NB, Scholl BJ, Chun MM (2008) Babies and brains: habituation in infant cognition and functional neuroimaging. Front Hum Neurosci 2:16. CrossRef Medline

Van Boxtel GJ (1998) Computational and statistical methods for analyzing event-related potential data. Behave Res Methods Instr Comp 30:87-102. CrossRef 